\title{
Der steife Ellenbogen: Fixateur-gestützte Distraktionsarthrolyse
}

\author{
Dietmar Pennig, Steffen Heck, Sascha Gick
}

\section{Zusammenfassung}

Ursächlich für die Ellenbogensteife sind häufig Trauma, Verbrennungen oder Koma. Eine Bewegungseinschränkung lässt sich gerade am Ellenbogen gelegentlich schwer verhindern und die Therapie ist anspruchsvoll. Neben einer detaillierten, individualisierten Analyse der Entstehung der Bewegungseinschränkung ist die genaue diagnostische Aufarbeitung vor jeder chirurgischen Intervention wichtig. Ellenbogensteife wird definiert als Streckdefizit von mehr als $30^{\circ}$ oder Beugung im Humeroulnargelenk von weniger als $130^{\circ}$. Die meisten Aktivitäten des täglichen Lebens spielen sich in einem Bewegungsausmaß von $100^{\circ}$ ab. Eingeschränkte Compliance, schlecht eingestellter Diabetes mellitus, Hepatitis-Bund -C-Infektionen, HIV-Infektionen sowie akute Gelenkinfektionen stellen
Kontraindikationen für die Distraktionsarthrolyse dar. Die geschlossene Distraktion des Ellenbogengelenks mit Fixateur externe (Arthrodiatasis) wird vorgestellt und bewertet. Die Arthrolyse des Ellenbogengelenks ist technisch aufwendig. Wenn allerdings Indikation und Verfahren richtig angewendet werden und Operateur, Physiotherapeut und nicht zuletzt der Patient mit dem Verfahren und der Nachbehandlung vertraut sind, lassen sich gute Langzeitergebnisse erzielen.

\section{Elbow Stiffness: Distraction Arthroplasty and External Fixation}

Loss of motion of the elbow is not uncommon after trauma, burns or coma and severely impairs upper limb function. Loss of motion may be difficult to avoid and is challenging to treat. Detailed analysis of the aetiology and di- agnostic evaluation is of the utmost importance for the planning of any surgical intervention for elbow stiffness. Stiff elbow is usually defined as less than $30^{\circ}$ in extension or as less than $130^{\circ}$ flexion. Most activities of daily living are possible if the elbow has a range of motion of $100^{\circ}\left(30-130^{\circ}\right.$ of flexion, Morrey's arc of motion). Contraindications are poor compliance, poorly controlled diabetes mellitus, active hepatitis B and C infection, HIV infection, acute articular infection. Current operative techniques, such as closed distraction with external fixation (arthrodiatasis), are presented and evaluated. Elbow arthrolysis is a technically demanding procedure. If indications and techniques are applied correctly and surgeon, physiotherapist, and patient are familiar with the procedure, good long-term results may be achieved.

\section{Einleitung}

Zur besseren Unterscheidung ist von einem residualen Beuge- bzw. Streckdefizit zu sprechen, auch Kombinationen werden regelhaft angetroffen. Die Gelenksteife wird nach nahezu allen Verletzungsmustern in Gelenknähe in einem bestimmten Prozentsatz beobachtet und lässt sich nicht zwingend einer speziellen Verletzungsart zuordnen. In der Literatur wird deutlich, dass neben dem speziellen Verletzungsmuster bzw. der Art der operativen Versorgung die Zeitdauer der nachfolgenden Gelenkimmobilisation für die Einsteifung verantwortlich ist.

Funktionell von größerer Bedeutung ist die Behinderung des Beugeumfangs.

OP-JOURNAL 2012; 28: 58-64

(c) Georg Thieme Verlag KG Stuttgart · New York DOI http://dx.doi.org/10.1055/s-0031-1298586
Während ein Streckdefizit bis zu einem gewissen Grade vorwiegend ein kosmetisches Problem darstellt, funktionell aber durch die Bewegung des Rumpfes ausgeglichen werden kann, steht für das Heranführen der Hand an den Körper keine Ausgleichsbewegung zur Verfügung. Selbst alltägliche Verrichtungen wie Körperpflege und Nahrungsaufnahme sind damit erheblich gestört.

Die in jüngerer Zeit entwickelten Techniken zum prothetischen Gelenkersatz am Ellbogengelenk sind mit Problemen belastet, sodass die Maxime, das natürliche Gelenk zu rekonstruieren, für die posttraumatischen Fälle nicht aufgegeben werden sollte [2].

Wichtig ist das Zeitintervall zwischen Unfallereignis und Operation in Bezug auf das Langzeitergebnis. Eine zunehmende Bewegungseinschränkung trotz adäquater Therapie macht einen recht- zeitigen Wechsel der Behandlungsstrategie erforderlich, ansonsten sind ungünstige Langzeitergebnisse zu erwarten [4].

\section{Pathophysiologie der Gelenksteife}

Im Falle lang andauernder Gipsimmobilisation ohne zusätzliche Verletzung des Kapselbandapparats tritt regelhaft eine Schrumpfung des Bandapparats auf. Eine gleichzeitige Zerreißung oder Teilzerstörung der Bänder mit nachfolgender Ausbildung von Narbengewebe verstärkt die Problematik jedoch erheblich.

Zusätzlich kommt es dabei zur Ausbildung von bandähnlichem, kollagenem Bindegewebe, das in Form von ungeordneten Narbensträngen die natürliche Bewegung in allen verletzten Bereichen des Gelenks hemmt. Als Ursache dafür kann der durch Schrumpfung des Kapselbandapparats oder des Narbengewebes hervorgerufene, kontinuierlich erhöhte Anpressdruck der humeroulnaren 
und der humeroradialen Gelenkflächen angenommen werden [16].

Dies führt zwangsläufig zu einem Verlust von Proteoglykanen aus der knorpeligen Grundsubstanz der Gelenkflächen, was sich radiologisch in einer Verschmälerung des sichtbaren Gelenkspalts widerspiegelt $[8,11,13,15]$.

\section{Operationsprinzip und -ziel}

Wiederherstellung des funktionellen Bewegungsumfangs nach Morrey von $100^{\circ}$.

Der Schwerpunkt der Behandlung liegt auf der Verbesserung der Beugefähigkeit.

- Behandlung der Unterarmdrehsteife zur Verbesserung von Pronation und Supination.

- Fixateur-gestütztes Verfahren zur Unterstützung der Physiotherapie mit Quengelelementen zur Verbesserung von Beugung und Streckung.

Das Verfahren der Distraktionsarthrolyse ist grundsätzlich für Patienten geeignet, die von der Compliance her einer Fixateurbehandlung zugeführt werden können.

Patienten mit schlecht kontrolliertem Diabetes mellitus, Hepatitis und HIV-Infektionen sind aus grundsätzlichen Erwägungen nicht geeignet, eine Fixateurbehandlung vornehmen zu lassen. Hier sind Pintrackinfektionen zu befürchten.

\section{Vorteile}

- Gedecktes Verfahren mit deutlich reduzierter Invasivität.

- Sichere Beurteilung des N. ulnaris bei zunehmender Flexion.

- Quengelungsmechanismus zur Vervollständigung der Flexion und Extension.

- Erleichterung der physiotherapeutischen Weiterbehandlung.

\section{Nachteile}

- Externes Verfahren.

- Nicht für alle Patienten geeignet.

- Risiko einer Pininfektion.

\section{Indikationen}

- Gesamtbewegungsausmaß von unter $100^{\circ}$ bei Vorliegen eines Flexionsdefizits.

- Streckdefizit von mehr als $30^{\circ}$.
- Kombination von Streck- und Beugedefiziten mit einer eingeschränkten Unterarmdrehung.

- Vorliegen heterotoper Ossifikationen im Gleitweg des Gelenks [6].

- Eingeschränkte Beweglichkeit des Ellenbogengelenks in Kombination mit reduzierter Beweglichkeit des Schultergelenks der gleichen Seite.

- Persistierende Luxations- und Subluxationsstellung im Ellenbogengelenk.

\section{Kontraindikationen}

- Patienten mit eingeschränkter Compliance.

- Schlecht eingestellter Diabetes mellitus.

- Hepatitis-B- und -C-Infektionen.

- HIV-Infektionen.

- Akute Gelenkinfektionen.

\section{Patientenaufklärung}

- Allgemeine Operationsrisiken wie Infektion, Thrombose, Embolie, Gefäßund Nervenschäden.

- Spezielle Risiken:

- Pininfektionen.

- Frakturen im Bereich der Pinstellen.

- Abriss von Knochenschalen/-fragmenten.

- Schädigung von Nerven durch verstärkte Flexion (N. ulnaris).

- Schädigung von Nerven durch verstärkte Extension (N. radialis und N. medianus).

- Schädigung von Gefäßen durch verstärkte Extension (A. brachialis).

- Verlust der Beweglichkeit.

- Nebenwirkungen von Indometacin zur Prophylaxe der heterotopen Ossifikation.

- Mechanische Beeinträchtigung des Distraktionsfixateurs.

- Revisionsoperationen.

\section{Operationsvorbereitungen (Tab. 1 und 2)}

- Ausschöpfen der physikalischen/therapeutischen Maßnahmen.

- Bei bestehender Kontraindikation gegen die Verabreichung von Indometacin Bestrahlung des Operationsgebiets.

- Kontakt mit anderen Patienten und gleichaltriger Behandlungstechnik.

- Computertomografie (CT) in 1-mmSchichten mit sagittaler und frontaler Rekonstruktion.
Tab. 1 Posttraumatische Ellenbogensteife.

\begin{tabular}{|c|c|}
\hline Präoperati & Maßnahmen \\
\hline Anamnese & \\
\hline $\begin{array}{l}\text { Röntgen- } \\
\text { verlauf }\end{array}$ & $\begin{array}{l}\text { - Unfallbilder, Versor- } \\
\text { gung, aktuelle Situation }\end{array}$ \\
\hline Diagnostik & - klinische Untersuchung \\
\hline & $\begin{array}{l}\text { - konventionelle } \\
\text { Röntgenbilder }\end{array}$ \\
\hline & - Arthrografie \\
\hline & - Spiral-CT \\
\hline & - EMG/NLG \\
\hline & $\begin{array}{l}\text { - Messblatt obere } \\
\text { Extremität }\end{array}$ \\
\hline Postoperat & e Maßnahmen \\
\hline Physiothera & e/Patientenkontakt \\
\hline Indometaci & $\times 50 \mathrm{mg}$ \\
\hline
\end{tabular}

- Neurologischer Untersuchungsbefund mit Messung der Nervenleitgeschwindigkeit.

- Gegebenenfalls Magnetresonanztomografie.

\section{Instrumentarium und Implantate}

- Bewegungsfixateur Ellenbogen und Standardfixateur (Orthofix S. R. L., Bussolengo, Italien).

- Allgemeines Knochen- und Gelenkinstrumentarium.

\section{Anästhesie und Lagerung}

- Allgemeinanästhesie ohne Plexuskatheter oder Plexusanästhesie.

- Standardoperationstisch mit Handtisch.

- Keine Blutleere.

\section{Operationstechnik (Abb. 1 bis 7)}

Distraktionsarthrolyse [3, 4, 12], (Tab. 3)

\section{Adjuvante operative Maßnahmen}

Die Dekompression des N. ulnaris ist beim Flexionsdefizit der 1 . operative Schritt und ist bereits oben erwähnt worden.

Das Dünnschicht-CT macht wesentliche Aussagen darüber, ob das Olekranon bzw. der Processus coronoideus in die 
Tab. 2 Diagnostik Ellenbogensteife.

\begin{tabular}{|c|c|}
\hline Verfahren & Bedeutung \\
\hline Unfallröntgenbilder & Ausmaß der Gelenkschädigung \\
\hline $\begin{array}{l}\text { Operative Versorgung } \\
\text { (Operationsberichte) } \\
\text { Röntgen }\end{array}$ & $\begin{array}{l}\text { Qualität der Reposition, Gelenkinkongruenz, Knorpel- } \\
\text { schäden? }\end{array}$ \\
\hline Konventionelles Röntgen & $\begin{array}{l}\text { Gelenkkongruenz, Knochenqualität Implantatlage und } \\
\text { Fixationsqualität } \\
\text { Osteophyten, Olekranon/Processus coronoideus }\end{array}$ \\
\hline CT (1-mm-Schichten) & $\begin{array}{l}\text { Humeroulnare (Sub-)Luxation } \\
\text { Intraartikuläre Fehlstellung } \\
\text { Osteophyten (Olekranon/Processus coronoideus) } \\
\text { Ausmauerung der Fossa olecrani/Fossa coronoidea } \\
\text { Lage der heterotopen Ossifikationen }\end{array}$ \\
\hline Arthrografie/Arthro-CT & $\begin{array}{l}\text { Fibrosierung der Gelenkkapsel } \\
\text { Ausmauerung des proximalen Radioulnargelenks }\end{array}$ \\
\hline MRT & $\begin{array}{l}\text { Beurteilung der Gelenkknorpeldichten und der Band- } \\
\text { strukturen } \\
\text { Lage der Nerven }\end{array}$ \\
\hline $\begin{array}{l}\text { NLG N. ulnaris, N. medianus und } \\
\text { N. radialis }\end{array}$ & Evaluation einer möglichen Vorschädigung \\
\hline
\end{tabular}

CT: Computertomografie; MRT: Magnetresonanztomografie; NLG: Nervenleitgeschwindigkeit

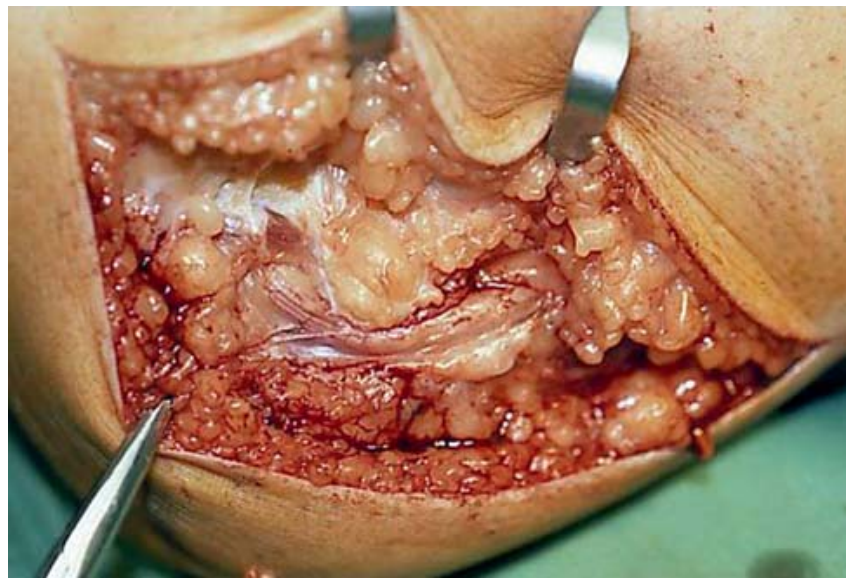

Abb. 1 Abbildung des linken Ellenbogens von ulnar. Nach leicht geschwungener Inzision wird der N. ulnaris dargestellt und in situ zwischen der Struthers-Arkade und der OsborneFaszie neurolysiert.

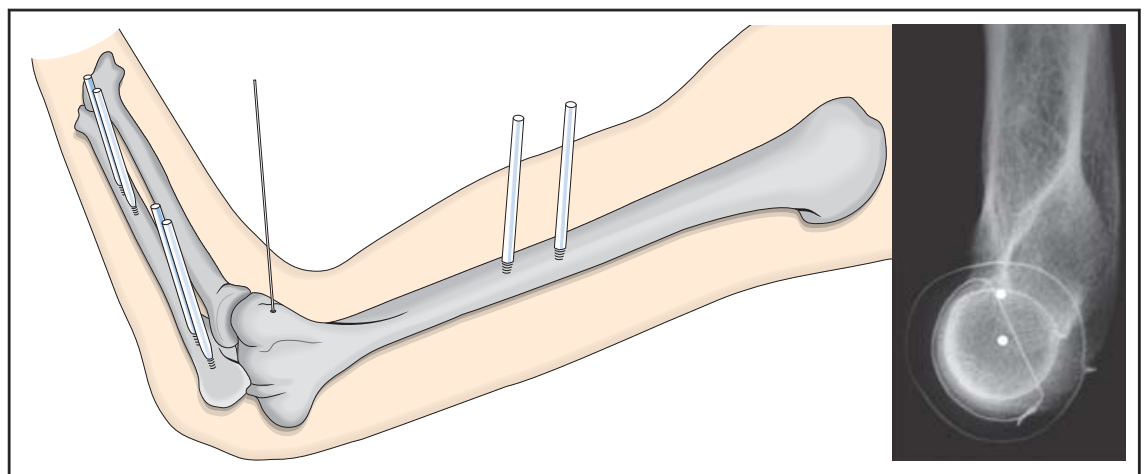

Abb. 2 Seitliches Röntgenschema zur Positionierung des Ziel-Kirschner-Drahts ( $2 \mathrm{~mm}$ ) kranial (oberer Punkt) des Bewegungszentrums (unterer Punkt). Metalldrähte wurden zur Markierung der 3 Trochleaanteile verwendet. Schematische Darstellung der Pinplatzierung ulnar und humeral; Positionierung des Zieldrahts im Drehzentrum des Ellenbogens. korrespondierende Fossa eintauchen kann.

Liegt eine Ausmauerung durch heterotopes Knochenmaterial vor, ist eine Entfernung dieses Materials vorzunehmen. In geeigneten Fällen kann zur Behandlung der Ausmauerung der Fossa olecrani und der Kontaktfläche des Processus coronoideus mit einer Hohlfräse ein gemeinsamer Raum für Olekranon und Processus coronoideus erzeugt und die normalerweise dünne Knochenlamelle der Fossa olecrani komplett entnommen werden.

Eine humeroulnare Subluxation in sagittaler Richtung, aber auch als Rotationsfehlstellung lässt sich über die Rekonstruktion des Dünnschicht-CT erkennen.

Liegt eine solche Subluxation vor, ist nach der Ursache zu suchen. Bei fehlverheilter Radiuskopffraktur oder fehlangeheiltem Processus coronoideus ist über entsprechende Osteotomien und Osteosynthesen die Gelenkkongruenz nach Distraktion wieder herzustellen.

In einzelnen Fällen kann eine allmähliche Distraktion des Gelenks, insbesondere bei dorsaler Subluxation, notwendig sein, um das Humeroulnargelenk korrekt einzustellen.

In einer 2. Maßnahme erfolgt dann die Wiederherstellung des Processus coronoideus durch einen Knochenblock.

In situ befindliches Osteosynthesematerial muss hinsichtlich der Störung der Gelenkbewegung beurteilt werden. In Bezug auf die Entfernung ist defensiv vorzugehen, eine vollständige Metallentfernung ist regelhaft nicht anzustreben.

Es sollte nur das Osteosynthesematerial entfernt werden, welches einer korrekten Einrichtung und normalen Bewegung des Humeroulnargelenks im Wege steht.

Eine routinemäßige Entfernung des Radiuskopfs zur Verbesserung der Beugung und Streckung des Ellenbogengelenks sollte nach unserer Auffassung nicht durchgeführt werden. Lediglich ein aufgrund der Verletzung zerstörter und nicht in eine kongruente Position $\mathrm{zu}$ bringender Radiuskopf kann reseziert werden. Inwieweit der endoprothetische Ersatz insbesondere beim jüngeren $\mathrm{Pa}$ tienten eine sinnvolle Alternative darstellt, bleibt abzuwarten, da Langzeitergebnisse noch nicht vorliegen. 

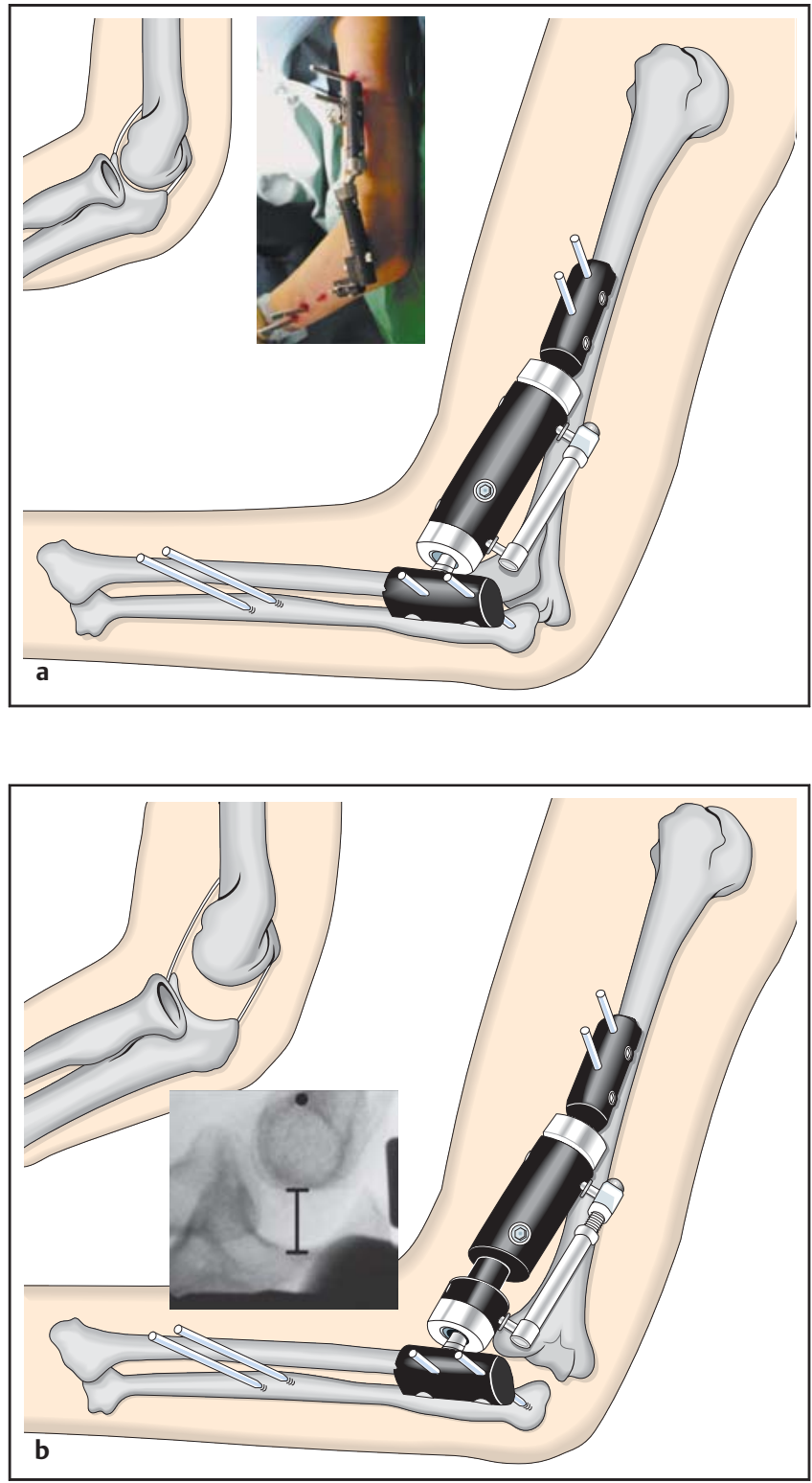

Abb. 3 b

Distraktion des Humeroulnargelenks mit Darstellung der verlängerten KapselBand-Strukturen (oberes kleines Bild). Das Röntgenschema zeigt die Separation der Gelenkflächen nach einer Distraktion von $15 \mathrm{~mm}$.

Die Konsequenz der Resektion des Radiuskopfs ist eine Destabilisierung des radialen Pfeilers mit entsprechender Beanspruchung der ulnaren Kollateralbandstrukturen. Erhalt und Rekonstruktion des Radiuskopfs (auch eines Teiles des Radiuskopfs) sollten immer vor einer Resektion stehen.

Arthroskopische Zusatznahmen am kontrakten Ellenbogen sind ausgesprochen schwierig, da der Gelenkinnenraum deutlich verkleinert ist und die Gelenkflächen aufeinandersitzen.

Das Risiko einer iatrogenen Knorpelverletzung oder einer Verletzung anderer Strukturen in Gelenknähe ist nicht unerheblich. Nach vorgenommener Distraktion des Gelenks mit Erweiterung des
Gelenkspalts ist jedoch die Einführung eines Arthroskops möglich. Aufgrund der lateralen Lage des Fixateurs ist die Auswahl der Portale jedoch eingeschränkt.

\section{Postoperative Behandlung}

Es ist sinnvoll, die dieser Behandlung unterzogenen Patienten in eine gemeinsame Sprechstunde einzubestellen und auch gemeinsam in Bezug auf die Nachbehandlung zu betreuen.

Die Physiotherapie kann mithilfe der Kompressions- und Distraktionselemente eine zunehmende Flexion erzeugen und ebenso die Extension verbessern. In Abhängigkeit vom Bewegungswiderstand ist ein Arretieren des Fixateurs al- ternierend in Beuge- und Streckstellung über Nacht sinnvoll.

Die Pinpflege sollte einmal wöchentlich durchgeführt werden; hierzu sind nicht färbende, milde Desinfektionsmittel empfehlenswert.

\section{Fehler, Gefahren, Komplikationen}

Eine vollständige präoperative Röntgendiagnostik inklusive 1-mm-CE-Schichten mit Rekonstruktion in frontaler und sagittaler Ebene ist unverzichtbar. Heterotope Ossifikationen im Gleitweg des Olekranons, des Processus coronoideus und des Radiuskopfs müssen beseitigt werden. Verbliebenes Osteosynthesematerial kann die Trochlea überdecken und zu Schwierigkeiten bei der Einbringung des Ziel-Kirschner-Drahts führen. Nicht korrekte Platzierung der ulnaren Pins kann zur insuffizienten Verankerung führen. Nicht korrekte Implantation der Olekranonpins kann zum Ausbruch unter Distraktionsbedingungen führen. Unvollständige Dekompression und Neurolyse des N. ulnaris können bei zunehmender Beugung eine Dehnung des Nervs mit Schädigung hervorrufen. Inkomplette Distraktion führt zu einer ungenügenden Separation der Gelenkflächen und einer nicht ausreichenden Dehnung der fibrosierten Kapsel-BandStrukturen. Ein ausgeprägtes Streckdefizit darf intraoperativ nicht vollständig ausgeglichen werden, da Überdehnung von neurovaskulären Strukturen auf der Beugeseite droht. Bei Fehlplatzierungen und Mehrfachbohrungen von Fixateurpins in Oberarm und Ulna kann es zu Frakturen in diesem Bereich kommen. Bei unzureichender Pinpflege droht ein Pinstelleninfekt. Nicht konsequent oder über einen zu kurzen Zeitraum (< 1 Jahr) durchgeführte Krankengymnastik zeigt ein unbefriedigendes Ergebnis und kann in einer neuerlichen Einsteifung resultieren.

Ergebnisse

Bei Kindern und Adoleszenten sind regelhaft posttraumatische Veränderungen die Ursache für eine Ellenbogensteife. Diese umfassen neben Frakturfolgen auch heterotope Ossifikationen nach Schädel-Hirn-Trauma und Kontrakturen nach Verbrennungen. Die präoperative Aufarbeitung der Entstehung der Ellenbogensteife entspricht der oben dargelegten Vorgehensweise. Auch hier ist die Computertomografie mit Rekonstruktion in der Frontal- und Sagittalebene von 

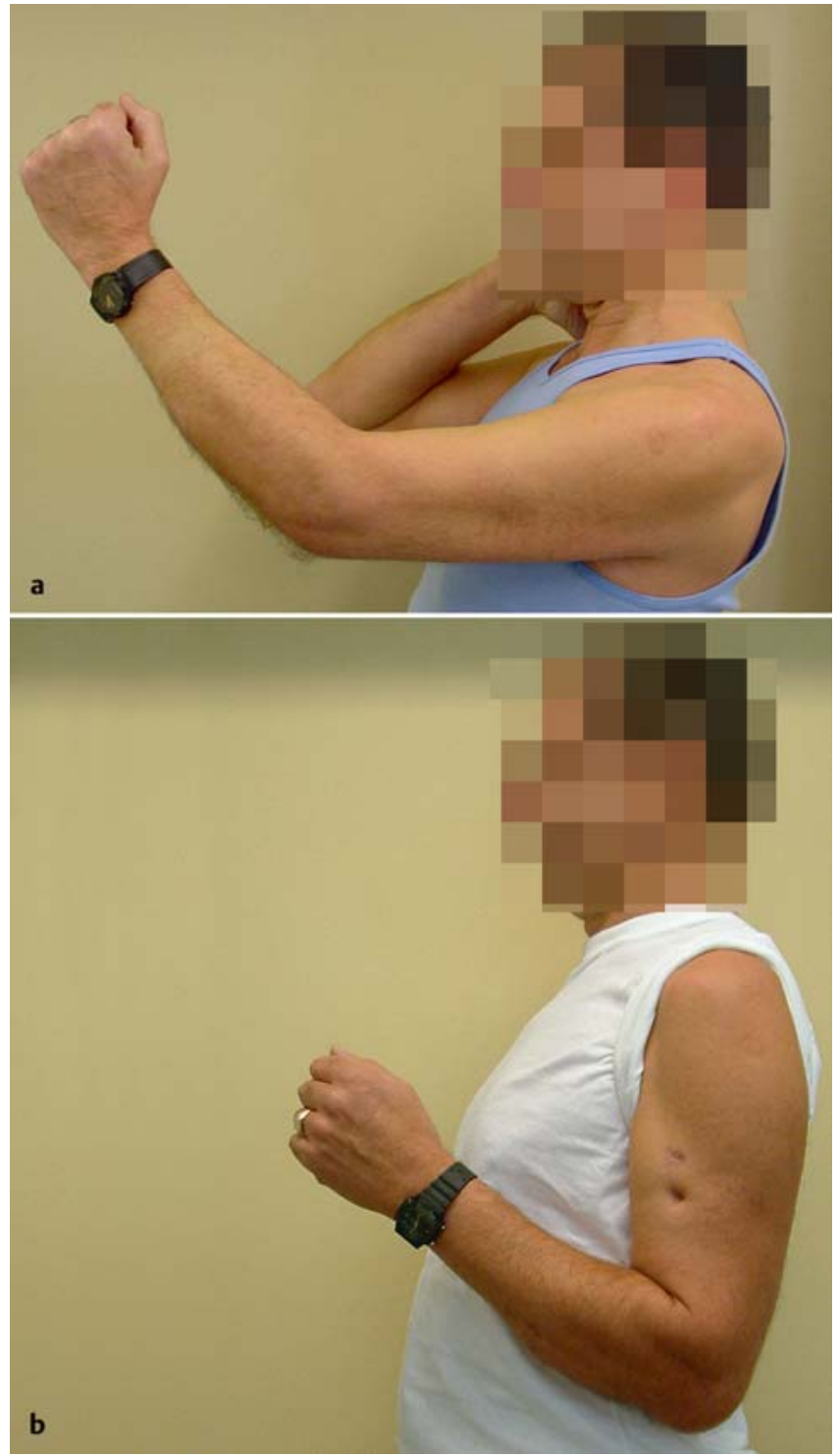

entscheidender Bedeutung. Die Durchmesser der Fixateurpins und der Typ des Distraktionsfixateurs müssen den kindlichen Skelettverhältnissen angepasst werden [9]. Bei einem negativen elektrophysiologischen Untersuchungsbefund der Nerven wird nur bei Kindern über 13 Jahren eine Dekompression des N. ulnaris routinemäßig vorgenommen [9]. Die Verweildauer des Fixateurs in dieser Altersgruppe beträgt insgesamt 6 Wochen.

Die Behandlungsergebnisse bei einer Nachuntersuchungszeit von 5 Jahren ergaben bei 14 Kindern und Heranwachsenden eine Verbesserung des präoperativen Bewegungsumfangs von $37^{\circ}$ in Bezug auf Extension und Flexion auf $108^{\circ}\left(75-130^{\circ}\right)$.
Abb. 4 a und b

Prä- und postoperative Beugung des linken Ellenbogens bei einem 45-jährigen Rechtshänder 8 Wochen nach Arthrodiatasis.

Im Vergleich mit den in der Literatur publizierten kleinen Serien nach offener Arthrolyse und Bewegungsschienennachbehandlung sind unsere Ergebnisse positiver $\mathrm{zu}$ bewerten. Eine besondere Herausforderung im Kindesalter stellt die notwendige krankengymnastische Übungsbehandlung dar. Die Einbeziehung der Erziehungsberechtigten ist hier von entscheidender Bedeutung für den Behandlungserfolg.

Ein Vorschlag zur Klassifikation der Ellenbogensteife findet sich in Tab.4. Das Ellenbogengelenk als zentrales Bewegungselement der oberen Gliedmaße ist von großer Bedeutung für die Positionierung des Greifinstruments „Hand“. Die Wiederherstellung der Ellenbogenfunktion nach Einsteifung ist eine anspruchsvolle Aufgabe. Die Aufarbeitung des Behandlungsverlaufs ist ebenso wie eine differenzierte präoperative Diagnostik und das Beachten der Operationstechnik als unabdingbare Voraussetzung für die Wiederherstellung der Ellenbogenfunktion zu sehen. Abzuzielen ist auf die Wiederherstellung des „functional arc“ nach Morrey $\left(100^{\circ}\right)$. Gekoppelt mit dieser Bewegung im Humeroulnargelenk muss eine Unterarmdrehung von $50^{\circ}$ in Form von Pro- und Supination vorhanden sein, um 90\% der täglichen Verrichtungen durchführen zu können $[9,10]$.

Nach Durchführung arthroskopischer und offener Arthrolysen ist die Wiedereinsteifung als Hauptkomplikation $\mathrm{zu}$ begreifen [1]. Eine Instabilität wird trotz Durchtrennung von Kapselbandstrukturen nicht beobachtet $[2,5]$. Prognostisch ungünstig sind nach unserer Erfahrung Narkosemanipulationen, die zu einer weitergehenden Schädigung der vulnerablen Gelenkflächen führen können.

Itoh [7] konnte eindrucksvoll demonstrieren, dass die Narkosemobilisation zur Behandlung der Ellenbogensteife nicht geeignet ist.

Die offene Arthrolyse wie von Morrey [9] und Sojbjerg beschrieben, stellt sicherlich eine Alternative zur Distraktionsarthroplastik dar $[13,15]$.

Es steht jedoch zu befürchten, dass der operative Eingriff aufgrund des erheblichen Weichteiltraumas zu einer neuerlichen Fibrosierung mit Beeinträchtigung der Gleitschichten führt. Wir halten die Distraktion des Ellenbogengelenkes mit daraus resultierender Separation der Gelenkflächen durch Verlängerung der geschrumpften Kapselbandstrukturen für einen physiologischen Weg, das operative Trauma zu reduzieren $[8,10,12,14]$.

Bedeutsam ist die Auswirkung von Flexions- und Extensionsdefiziten auf die Funktion der das Ellenbogengelenk kreuzenden Nerven.

Insbesondere der $\mathrm{N}$. ulnaris in seinem osteofibrösen Kanal ist aufgrund der engen topografischen Beziehung zum Gelenk gefährdet.

Die Dekompression und Neurolyse dieses Nervs ist dementsprechend regelmäßig durchzuführen, eine Verlegung des osteofibrösen Kanals mit heterotopem Knochen- oder Osteosynthesematerial muss begleitend therapiert werden. Eine Transposition des Nervs im Sinne der Ventralisierung wird von uns nicht emp- 


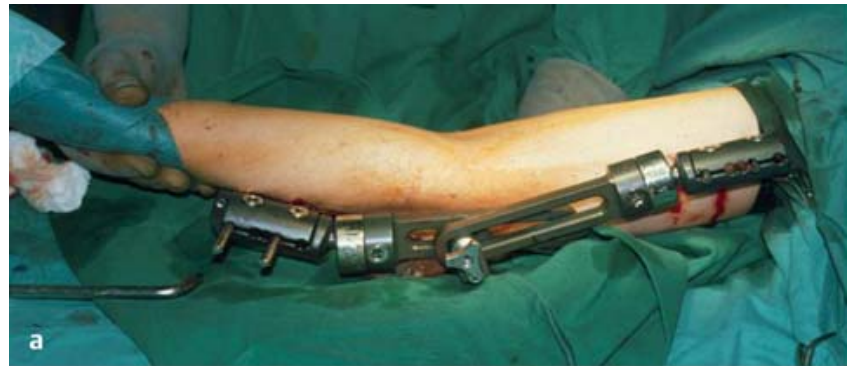

Abb. 5 a und $\mathbf{b}$ Intraoperative Streckung (a) und Beugung (b) im Bewegungsfixateur unter Distraktion ohne zusätzliche chirurgische Weichteilmaßnahmen.

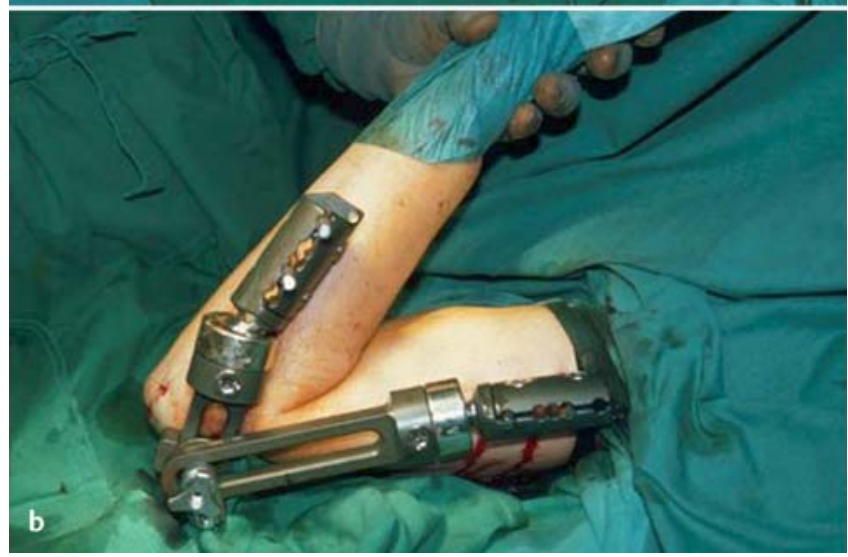

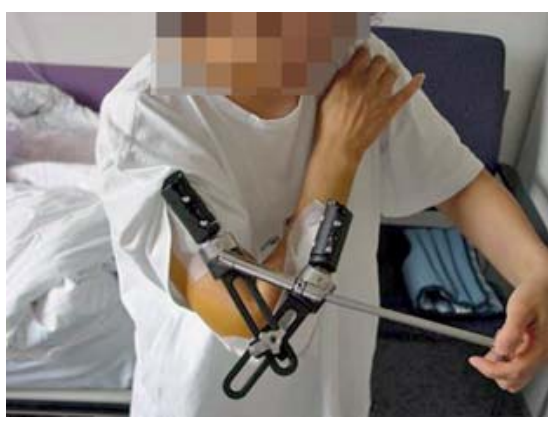

Abb. 6 Demonstration der KompressionsDistraktions-Einheit zur postoperativen Verbesserung der Beugung unter Distraktion. Der umgekehrte Einsatz dient zur Verbesserung der Streckung. fohlen, da wir um die Funktion des minderdurchbluteten und im vernarbtem ventralen Gewebe gelegenen Nervs fürchten. Lediglich bei einer pathologischen Anspannung der Nerven nach Durchführung der Distraktion ist die Transposition zu erwägen.

Bei länger bestehenden Extensionsdefiziten kommt es zur Verkürzung der ventral gelegenen Nerven und Gefäße.

Obwohl es nach Distraktion möglich ist, die Streckung vollständig herbeizuführen, ist hier Vorsicht geboten. Die zu erreichende Extension sollte graduell mithilfe der dafür vorgesehenen Distraktoren geschehen, um eine allmähliche Anpassung des Gewebes analog dem

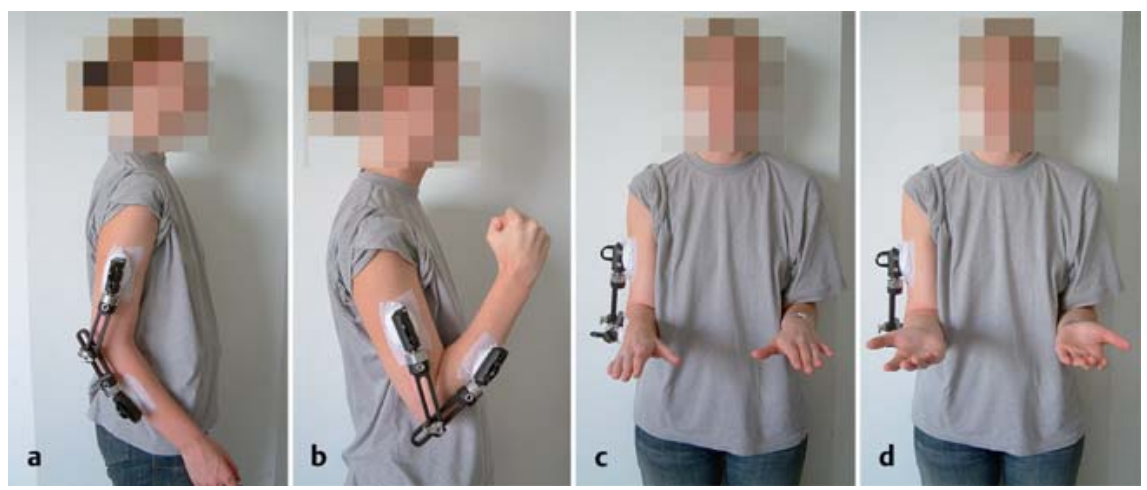

Abb. 7 a bis $\mathbf{d}$ a und $\mathbf{b}$ Postoperativer Bewegungsumfang 10 Tage nach Arthrodiatasis mit präoperativer Ellenbogensteife. $\mathbf{c}$ und $\mathbf{d}$ Die Unterarmdrehung bleibt durch den anliegenden Bewegungsfixateur unbeeinträchtigt.
Tab. 3 Zusammenfassung.

\begin{tabular}{ll}
$\begin{array}{l}\text { Intraoperative } \\
\text { Distraktion }\end{array}$ & $\begin{array}{l}\text { Distraktionsfixa- } \\
\text { teur: } 2 \times 15 \mathrm{~mm}\end{array}$ \\
\hline Relaxationsphase & 6-10 Tage \\
\hline Mobilisationsphase & $\begin{array}{l}\text { unter Distraktion } \\
\text { (5-6 Wochen) }\end{array}$
\end{tabular}

\begin{tabular}{|c|c|}
\hline Typ 1 & $\begin{array}{l}\text { Gelenk kongruent, keine } \\
\text { heterotopen Ossifikationen }\end{array}$ \\
\hline Typ 2 & $\begin{array}{l}\text { Gelenk kongruent, hetero- } \\
\text { tope Ossifikationen (CT) }\end{array}$ \\
\hline Тур 3 & $\begin{array}{l}\text { Gelenk (sub)luxiert, keine } \\
\text { heterotopen Ossifikationen }\end{array}$ \\
\hline Typ 4 & $\begin{array}{l}\text { Gelenk (sub)luxiert, hete- } \\
\text { rotope Ossifikationen }(\mathrm{CT})\end{array}$ \\
\hline Typ 5 & $\begin{array}{l}\text { Gelenk inkongruent, keine } \\
\text { heterotopen Ossifikationen }\end{array}$ \\
\hline Typ 6 & $\begin{array}{l}\text { Gelenk inkongruent, hete- } \\
\text { rotope Ossifikationen (CT) }\end{array}$ \\
\hline Тур 7 & Ankylose \\
\hline
\end{tabular}

CT: Computertomografie

Vorgehen der Gliedmaßenverlängerung zu erzielen. Es hat sich gezeigt, dass ca. die Hälfte des vorhandenen Extensionsdefizits intraoperativ ausgeglichen werden kann, der verbleibende Rest über einen Zeitraum von etwa 10 Tagen mithilfe der mechanischen Hilfsmittel (Quengeldistraktoren). Die Distraktionsgeschwindigkeit sollte $4 \mathrm{~mm}$ pro Tag nicht überschreiten. Nervenfunktion und Durchblutung müssen aufmerksam beobachtet werden.

Die Nachbehandlung sollte idealerweise in der operierenden Klinik vorgenommen werden.

Eine enge Anbindung der krankengymnastischen Übungsbehandlung ist als Vorteil zu sehen und ist integraler Teil des Behandlungskonzepts. Sie muss bereits vor Planung des Operationszeitpunkts in der ambulanten Nachsorge gesichert werden. Die Nachbehandlung zieht sich regelhaft über 1 Jahr, gelegentlich sogar über 2 Jahre hin. Wie bei allen Korrekturen unter Einsatz eines äußeren Festhalters hat sich das Gespräch des Betroffenen mit anderen in Behandlung befindlichen Patienten bewährt. 
Das nicht ärztliche Personal ist in der Nachbehandlung von besonderer Bedeutung.

Ohne die Einbeziehung von Pflegepersonal und Krankengymnastik ist der therapeutische Erfolg nicht zu realisieren.

\section{Interessenkonflikt}

Der korrespondierende Autor weist auf folgende Beziehung hin: Prof. Dr. Pennig erhält Lizenzgebühren der Fa. Orthofix S. R. L., Bussolengo/Italien.

\section{Literatur}

1 Breen TF, Gelbermann RH, Ackermann GN. Elbow flexion contractures. Treatment by anterior release and continuous passive motion. J Hand Surg (Br) 1988; 13: 286

2 Gates, HS, Sullivan FL, Urbaniak JR. Anterior capsulotomy and continuous passive motion in the treatment of post-traumatic flexion contracture of the elbow. J Bone Joint Surg (Am) 1992 74: 1229-1234

3 Gausepohl T, Koebke J, Pennig D et al. Anatomische Grundlagen zur Anwendung der unilateralen externen Fixation an Oberarm, Unterarm und Hand. Osteosyn Int 1997; 5: 76-88
${ }^{4}$ Gausepohl T, Mader K, Pennig D. Mechanical distraction for the treatment of posttraumatic stiffness of the elbow in children and adolescents. J Bone Joint Surg (Am) 2006; 88 A: 1011-1021

${ }^{5}$ Green DP, McCoy H. Turnbuckle orthotic correction of elbow flexion contractures after acute injuries. J Bone Joint Surg (Am) 1979; 61: 1092

${ }^{6}$ Ilahi OA, Bennett JB, Gabel GT et al. 3rd Classification of heterotopic ossification about the elbow. Orthopedics 2001; 24: 1075-1078

${ }^{7}$ Itoh Y, Saegusa K, Ishiguro T et al. Operation for the stiff elbow. Int Orthop 1989; 13: 263268

${ }^{8}$ Mader K, Pennig D, Gausepohl T, Wulke AP. Arthrolyse des Ellenbogengelenkes. Unfallchirurg 2004; 107: 403-414

${ }^{9}$ Morrey BF. Post-traumatic contracture of the elbow. J Bone Joint Surg (Am) 1990; 72: 601618

${ }^{10}$ Morrey BF. Distraction Arthroplasty. In: Morrey $\mathrm{BF}$ (ed). The Elbow. New York: Raven; 1994

11 Pennig D, Gausepohl T. Die posttraumatische Ellenbogensteife - Gelenkdistraktion mit Fixateur externe als Behandlungskonzept. In: Meyer RP, Kappeler U (Hrsg). Ellenbogenchirurgie in der Praxis. Berlin: Springer; 1998

12 Pennig D, Gausepohl T, Mader K, Wolfgarten B. Die Distraktionsarthrolyse mit humeroulnarem Bewegungsfixateur zur Behandlung der posttraumatischen Ellenbogensteife. Orthopäde 2001; 30: 635-644
13 Pennig D, Mader K, Gausepohl T. Bewegungseinschränkung nach Verletzung des Ellenbogengelenkes: Planung und operative Strategie der Arthrolyse. Zentralbl Chir 2005; 35: 65-71

${ }^{14}$ Regan WD, Reilly CD. Distraction arthroplasty of the elbow. Hand Clin 1993; 9: 719-728

${ }^{15}$ Weizenbluth M, Eichenblat M, Lipskeir E et al. Arthrolysis of the elbow: 13 cases of posttraumatic stiffness. Acta Orthop Scand 1989; 60: 642

${ }^{16}$ Wolff J. Über die Operation der Ellenbogengelenkankylose. Berliner Klinische Wochenschrift 1895; 43: 44

\section{Prof. Dr. med. Dietmar Pennig Chefarzt}

Dr. med. Steffen Heck

Ltd. Oberarzt

Dr. med. Sascha Gick

Oberarzt

Klinik für Unfallchirurgie/Orthopädie, Hand- und Wiederherstellungschirurgie

St. Vinzenz-Hospital

Merheimer Straße 221-223

50733 Köln

dietmar.pennig@vinzenz-hospital.de 